\title{
ZERO-DIMENSIONAL FAMILIES OF SETS ${ }^{1}$
}

\author{
SAMUEL EILENBERG AND E. W. MILLER
}

A family $\Phi=\left\{A_{\alpha}\right\}$ of subsets of a topological space $X$ will be called 0 -dimensional if given an open set $U$ such that $A_{\alpha_{0}} \subset U$, there is an open set $V$ such that (1) $A_{\alpha_{0}} \subset V \subset U$ and (2) $(\bar{V}-V) \sum_{\alpha} A_{\alpha}=0$. We enumerate below a few of the most common 0-dimensional families. In each case the proof of 0 -dimensionality is easy, and is therefore omitted.

(I) Every family of disjoint open subsets of a topological space is 0 -dimensional.

(II) Let $Y$ be a locally connected subset of a topological space $X$. The family $\Phi$ of the components of $Y$ is 0 -dimensional.

(III) Let $Y$ be a compact and closed subset of a metric space $X$. The family $\Phi$ of the components of $Y$ is 0 -dimensional.

(IV) Let $Y$ be a subset of a metric space $X$. The family $\Phi$ consisting of the individual points of $Y$ is 0 -dimensional if and only if $\operatorname{dim} Y=0$.

(V) Let $\Phi$ be a family of closed subsets of a compact metric space $X$. If, given any sequence $F, F_{1}, F_{2}, \cdots$ of sets of $\Phi$, the relation $F \cdot \lim \inf F_{i} \neq 0$ implies $\lim \inf F_{i} \subset F$, then the family $\Phi$ is called $u p-$ per-semi-continuous. In this case the sets of the family $\Phi$ are disjoint. There is a standard way of introducing a topology into the family $\Phi$ which leads to a separable metrizable hyperspace $\Phi^{*}$. The family $\Phi$ is 0 -dimensional if and only if $\operatorname{dim} \Phi^{*}=0$. In particular, $\Phi$ is 0 -dimensional whenever it is upper-semi-continuous and countable.

(VI) Let $Y$ be a subset of a topological space $X$ and let $Y$ be homeomorphic with a subset of the linear continuum. The family $\Phi$ of the components of $Y$ is 0 -dimensional.

The purpose of this note is to establish the following theorem:

Theorem. Let $X$ be a unicoherent Peano continuum, ${ }^{2} \Phi=\left\{A_{\alpha}\right\}$ a 0-dimensional family of subsets of $X$, and $x_{1}$ and $x_{2}$ two points of $X$. If none of the sets $A_{\alpha}$ cuts $X$ between $x_{1}$ and $x_{2},{ }^{3}$ then $\sum_{\alpha} A_{\alpha}$ does not cut $X$ between $x_{1}$ and $x_{2}$.

Various corollaries can be obtained by taking $X$ to be the $n$-sphere

\footnotetext{
1 Presented to the Society, December 26, 1939, under the title On 0-dimensional upper-semi-continuous collections.

${ }^{2}$ A Peano continuum $X$ is called unicoherent if given any decomposition $X=X_{1}+X_{2}$ into continua, the set $X_{1} \cdot X_{2}$ is a continuum.

${ }^{3}$ A set $A \subset X$ cuts $X$ between $x_{1}$ and $x_{2}$ if $X-A$ contains no continuum joining $x_{1}$ and $x_{2}$.
} 
$S^{n}(n>1)$ or the $n$-cube $Q^{n}(n>0)$ and $\Phi$ to be one of the families (I) $-(\mathrm{VI}) .^{4}$

We shall establish two lemmas before giving the proof of the theorem.

Lemma 1. Let $X$ be a unicoherent Peano continuum. Let $A_{1}$ and $A_{2}$ be open and disjoint subsets of $X$, and let $A_{2}$ be connected. If neither $A_{1}$ nor $A_{2}$ cuts $X$ between the points $x_{1}$ and $x_{2}$, then $A_{1}+A_{2}$ does not cut $X$ between $x_{1}$ and $x_{2}$.

Proof. Let $C_{2}$ be the component of $X-A_{2}$ which contains $x_{1}$ and $x_{2}$. Let $B_{1}=A_{1} \cdot C_{2}$ and $B_{2}=X-C_{2}$. It follows easily that $B_{1}$ and $B_{2}$ are open, $B_{1} \cdot B_{2}=0, B_{2}$ and $X-B_{2}=C_{2}$ are connected, and $A_{1}+A_{2}$ $C B_{1}+B_{2}$. It will be sufficient to show that $B_{1}+B_{2}$ does not cut $X$ between $x_{1}$ and $x_{2}$.

Since $B_{1} \subset A_{1}$, the set $B_{1}$ does not cut $X$ between $x_{1}$ and $x_{2}$. We will denote the component of $X-B_{1}$ which contains $x_{1}$ and $x_{2}$ by $C_{1}$. If $C_{1} \cdot B_{2}=0$, then $C_{1} \subset X-\left(B_{1}+B_{2}\right)$, so that $B_{1}+B_{2}$ does not cut $X$ between $x_{1}$ and $x_{2}$. We will suppose then that $C_{1} \cdot B_{2} \neq 0$. Since $C_{1}$ and $B_{2}$ are connected it follows that $C_{1}+B_{2}$ is connected. But $C_{1}+B_{2} \subset X-B_{1}$. Hence $C_{1}+B_{2} \subset C_{1}$, and therefore $B_{2} \subset C_{1}$. Since $C_{2}=X-B_{2}$, it follows that $X=C_{1}+C_{2}$. Since $C_{1}$ and $C_{2}$ are continua and $X$ is unicoherent, it follows that $C_{1} \cdot C_{2}$ is a continuum. But $x_{1}$ and $x_{2}$ belong to $C_{1} \cdot C_{2}$, and $C_{1} \cdot C_{2} \subset X-\left(B_{1}+B_{2}\right)$. Hence $B_{1}+B_{2}$ does not cut $X$ between $x_{1}$ and $x_{2}$.

LEMma 2. Let $X$ be a unicoherent Peano continuum and let $A_{1}, A_{2}, \cdots ; A_{n}, \cdots$ be a sequence of disjoint open subsets of $X$. If none of the sets $A_{n}$ cuts $X$ between $x_{1}$ and $x_{2}$, then $\sum_{n=1}^{\infty} A_{n}$ does not cut $X$ between $x_{1}$ and $x_{2}$.

Proof. Since $X$ is locally connected and separable, every open set in $X$ consists of a countable number of components each of which is open. It is clear, then, that we may assume each set $A_{n}$ to be connected.

Let $k$ be any positive integer. Using Lemma 1 , it follows by finite induction that $A_{1}+A_{2}+\cdots+A_{k}$ does not cut $X$ between $x_{1}$ and $x_{2}$. Let $C_{k}$ be the component of $X-\left(A_{1}+A_{2}+\cdots+A_{k}\right)$ which contains $x_{1}$ and $x_{2}$. Then $\coprod_{k=1}^{\infty} C_{k}$ is a continuum containing $x_{1}$ and $x_{2}$, and $\coprod_{k=1}^{\infty} C_{k} \subset X-\sum_{k=1}^{\infty} A_{k}$. Hence $\sum_{k=1}^{\infty} A_{k}$ does not cut $X$ between $x_{1}$ and $x_{2}$.

${ }^{4}$ See R. L. Moore, Proceedings of the National Academy of Sciences, vol. 10 (1934), p. 356, and S. Eilenberg, Fundamenta Mathematicae, vol. 26 (1936), pp. 7677. 
We now return to the proof of the theorem:

For any $F$ in $\Phi$ there is an open set $U(F)$ such that $F \subset U(F)$, and $U(F)$ does not cut $X$ between $x_{1}$ and $x_{2}$. Since $\Phi$ is 0 -dimensional, there is an open set $V(F)$ such that $F \subset V(F) \subset U(F)$ and $[\overline{V(F)}-V(F)] \sum F$ $=0$. By the Lindelöf covering theorem, there is a sequence $F_{1}, F_{2}, \ldots$. of elements of $\Phi$ such that $\sum F \subset \sum_{i=1}^{\infty} V\left(F_{i}\right)$. Now let

$$
\begin{aligned}
& A_{1}=V\left(F_{1}\right), \quad A_{2}=V\left(F_{2}\right)-\overline{V\left(F_{1}\right),} \\
& A_{k}=V\left(F_{k}\right)-\overline{\left[V\left(F_{1}\right)+\cdots+V\left(F_{k-1}\right)\right]}, \cdots .
\end{aligned}
$$

The sets $A_{1}, A_{2}, \cdots, A_{k}, \cdots$ are open and disjoint, and no one of them cuts $X$ between $x_{1}$ and $x_{2}$. But, as is easily shown, $\sum F \subset \sum_{k=1}^{\infty} A_{k}$. Hence in view of Lemma $2, \sum F$ does not cut $X$ between $x_{1}$ and $x_{2}$.

The University of Michigan

\section{SUMS OF FOURTH POWERS OF GAUSSIAN INTEGERS}

\section{IVAN NIVEN}

It is the purpose of this note to give necessary and sufficient conditions for the expressibility of a Gaussian integer as a sum of fourth powers of Gaussian integers; and then to determine an upper bound to the number of fourth powers necessary when the conditions are satisfied. Our results are as follows:

Theorem. A Gaussian integer is expressible as a sum of fourth powers of Gaussian integers if and only if its imaginary coordinate is divisible by 24 . Every integer $a+24 b i$, where $a$ and $b$ are rational integers, is expressible as a sum of 18 or fewer fourth powers.

First we prove that the condition is necessary. We note that ${ }^{1}$

$$
(x+y i)^{4}=x^{4}-6 x^{2} y^{2}+y^{4}+4 i x y\left(x^{2}-y^{2}\right) .
$$

It is obvious that $x y\left(x^{2}-y^{2}\right)$ is divisible by 2 and by 3 . Hence any fourth power has an imaginary coordinate divisible by 24 , and any sum of fourth powers has the same property.

The converse of this is included in the second statement in the theorem, which we now proceed to prove. The author ${ }^{2}$ has shown that a Gaussian integer $a+2 b i$ is expressible as a sum of two squares

\footnotetext{
1 Latin letters will represent rational integers throughout this paper.

${ }^{2}$ Integers of quadratic fields as sums of squares, Transactions of this Society, vol. 48 (1940), p. 410, Theorem 2.
} 